This is the submitted version (before peer-review) of the following article:

Jonauskaite, D. \& Mohr, C. (in press). A commentary: The sun is no fun without rain: Reply to "The sun and how do we feel about the color yellow? Methodological concerns". Journal of Environmental Psychology. https://doi.org/10.1016/j.jenvp.2019.101379

It has been published in final form at https://doi.org/10.1016/j.jenvp.2019.101379. This article may be used for non-commercial purposes under the CC-BY-NC-ND 4.0 license http://creativecommons.org/licenses/by-nc-nd/4.0/. 
A commentary: The sun is no fun without rain: Reply to "The sun and how do we feel about the color yellow? Methodological concerns"

Domicele Jonauskaite $^{1 *}$ and Christine Mohr $^{1}$

${ }^{1}$ Institute of Psychology, University of Lausanne, Switzerland

* Corresponding author: Institute of Psychology, University of Lausanne, Quartier Mouline, Bâtiment Géopolis, CH-1015, Lausanne, Switzerland, +41 21692 32 52, Domicele.Jonauskaite@unil.ch ORCID: 0000-0002-7513-9766 
Studying the link between colour and emotion, or colour and affect more widely, attracts interest and criticism from two major study domains that naturally speak little to each other, namely vision science and linguistics. In both domains, researchers represent their respective study domain frequently expressing strong opinions about the importance, if not sole importance, of their research fields and methodologies. Cognitive psychologists working on the affective meaning of colour will touch upon both domains; or rather find themselves right in-between. Thus, we are not surprised by the current comments, welcoming the interest and criticisms. We consider the commentary a valuable opportunity to communicate differences and highlight opportunities for inter-disciplinary attempts to answer shared questions using different methodologies.

In our study (Jonauskaite, Abdel-Khalek, et al., 2019), we replicated that yellow and joy are associated. Yellow-joy associations were previously detected with a variety of methods (Burkitt \& Sheppard, 2014; Dael, Perseguers, Marchand, Antonietti, \& Mohr, 2016; Jonauskaite, Althaus, Dael, Dan-Glauser, \& Mohr, 2019; Kaya \& Epps, 2004; Lindborg \& Friberg, 2015; Sutton \& Altarriba, 2016). We further reported that this association varied as a function of climatological factors in 55 countries. Participants living in countries farther away from the equator and/or with higher annual precipitation levels associated yellow with joy to a greater extent. We concluded that environmental experiences contribute to the affective meaning of colour. Here, we reply to the two concerns raised by Azer in his commentary (Azer, 2019), i) the variability in sample sizes and ii) the importance of colour metaphors.

\section{Sample size}

The author highlighted that our sample sizes varied substantially (i.e., from 20 Zimbabwean to 499 Greek participants). The question is whether this variability is a hazard to our conclusions. Our data come from an ongoing survey (Mohr, Jonauskaite, Dan-Glauser, Uusküla, \& Dael, 2018). Thus, whatever the study question, sample sizes will differ between countries (e.g., (Jonauskaite, Wicker, 
et al., 2019). In the actual study, we included as many countries as possible in order to have a large spread in our predictor variables, which were fixed per country. Based on a previous recommendation, we included a country if it had at least 20 participants (Simmons, Nelson, \& Simonsohn, 2011) and accounted for uneven population sizes using the hierarchical cumulative link mixed model (Christensen, 2018). Alternatively, we could have included fewer countries of larger sample sizes, or randomly chosen 20 participants from each of the 55 countries. Both options would lead to lower accuracy due to a reduced number of participants.

To test if certain countries or participants were determinant for our results, we supplemented our original analyses with bootstrapping (Efron, 1979). Bootstrapping provides information about the reliability of our predictive model by simulating what the model could have been if a different subsample of countries or participants had been studied. We created an algorithm that randomly resampled the original data $10^{\prime} 000$ times in a two-step procedure. The two-step procedure simulated our recruitment strategy. We first drew 55 countries with replacement, and then we drew 6625 participants with replacement. To each new resampled dataset, we fitted the model that best explained our data (Jonauskaite, Abdel-Khalek, et al., 2019, pages 4-5, Block 3). This bootstrapping estimated the distribution of the coefficients for each predictor variable and their respective $95 \%$ confidence intervals $(95 \% \mathrm{Cl})$. The $95 \% \mathrm{Cl}$ for absolute latitude was $[0.012,0.042]$ and for precipitation was $[0.033,1.020]$. Since the confidence intervals do not include 0 , these variables significantly predict the yellow-joy associations. The $95 \% \mathrm{Cl}$ for sunshine was $[-0.031,0.015]$ and includes 0 . Therefore, sunshine is not a significant predictor of yellow-joy associations. Overall, the same predictors were significant in bootstrapping and in our study.

\section{Colour metaphors}

The author also highlighted a lack of culture-specific explanations referring to colour metaphors. True, many languages have metaphors involving colour terms. Thus, we are not surprised to learn 
about two Egyptian metaphors - yellow as a lemon and yellow-eyed, which have negative connotations. The justified question is whether such metaphors would explain our findings that yellow is less joyful in some countries (e.g. Egypt) than others.

In our article, we described how climatological factors predicted yellow-joy associations. The mechanisms through which climatological factors impact these cognitive associations are unknown. The causality question was outside the scope of our study. In the discussion, we suggested that "yellow-joy associations [could] emerge because of an individual's experience (sunshine makes all colours more vibrant), physical sensations (the positive feeling of skin warmed by the sun), embodied experience (doing joyful things in the sunshine) or semantic pathways (talking about joyful things and sunshine together)" (page 5). Colour metaphors could be another mechanism linking climatological experiences with colour-emotion associations. The author mentioned two Egyptian examples and claimed that "these idiomatic expressions are unique to the Egyptian culture". While we can follow the overall argument, we do not agree that such metaphorical connotations of yellow are unique to the Egyptian language. We are aware of metaphors that include the term yellow and carry negative connotations such as yellow-bellied (to be cowardly) in English, Gelb vor Neid sein (to be envious) in German, or rire jaune (to laugh out of embarrassment) in French. In English, there seem to be no positive metaphors with yellow (Allan, 2009). Despite these negative metaphorical connotations, $55-75 \%$ of English, French and German speakers associated yellow with joy. Participants from any country, including Egypt, did not associate yellow with negative emotions (see Table S9 in Jonauskaite, Abdel-Khalek, et al., 2019). Therefore, speakers of different languages may possess negatively charged colour metaphors and still endorse positive emotion associations with respective colours.

To definitively understand the role of colour metaphors in yellow-joy associations, we would have needed to systematically analyse colour metaphors in 40 languages and 55 countries. Nonetheless, understanding the explanatory power of colour metaphors for colour-emotion associations is an 
important future direction (e.g., see Barchard, Grob, \& Roe, 2017). We provide our data for further use (https://forsbase.unil.ch/project/study-public-overview/15126/1672/) and hope that researchers from different backgrounds would take up the task to further explain inter-individual and inter-national variance(s) using other methodologies.

Acknowledgements: This research was supported by the Swiss National Science Foundation, providing a Doc.CH fellowship grant to DJ (POLAP1_175055) and a project funding grant to CM (100014_182138). We thank Cornelis B. Doorenbos for his support running the bootstrapping models. We also thank Jean-Philippe Antonietti for verifying the accuracy of our text in regards to the description of our statistical methods.

\section{References}

Allan, K. (2009). The connotations of English colour terms: Colour-based X-phemisms. Journal of Pragmatics, 41(3), 626-637. https://doi.org/10.1016/j.pragma.2008.06.004

Azer, S. (2019). The sun and how do we feel about the color yellow? Methodological concerns. Journal of Environmental Psychology.

Barchard, K. A., Grob, K. E., \& Roe, M. J. (2017). Is sadness blue? The problem of using figurative language for emotions on psychological tests. Behavior Research Methods, 49(2), 443-456. https://doi.org/10.3758/s13428-016-0713-5

Burkitt, E., \& Sheppard, L. (2014). Children's colour use to portray themselves and others with happy, sad and mixed emotion. Educational Psychology, 34(2), 231-251. https://doi.org/10.1080/01443410.2013.785059

Christensen, R. H. B. (2018). Ordinal - regression models for ordinal data. R package version 2018.825. Retrieved from http://www.cran.r-project.org/package=ordinal/ 
Dael, N., Perseguers, M.-N., Marchand, C., Antonietti, J.-P., \& Mohr, C. (2016). Put on that colour, it fits your emotion: Colour appropriateness as a function of expressed emotion. Quarterly Journal of Experimental Psychology, 69(8), 1619-1630.

https://doi.org/10.1080/17470218.2015.1090462

Efron, B. (1979). Bootstrap methods: Another look at the jackknife. The Annals of Statistics, 7(1), 126. https://doi.org/10.1214/aoms/1177692541

Jonauskaite, D., Abdel-Khalek, A. M., Abu-Akel, A., Al-Rasheed, A. S., Antonietti, J.-P., Ásgeirsson, Á. G., ... Mohr, C. (2019). The sun is no fun without rain: Physical environments affect how we feel about yellow across 55 countries. Journal of Environmental Psychology, 66, 101350. https://doi.org/10.1016/j.jenvp.2019.101350

Jonauskaite, D., Althaus, B., Dael, N., Dan-Glauser, E., \& Mohr, C. (2019). What color do you feel? Color choices are driven by mood. Color Research \& Application, 44(2), 272-284. https://doi.org/10.1002/col.22327

Jonauskaite, D., Wicker, J., Mohr, C., Dael, N., Havelka, J., Papadatou-Pastou, M., ... Oberfeld, D. (2019). A machine learning approach to quantify the specificity of colour-emotion associations and their cultural differences. Royal Society Open Science, 6(9), 190741. https://doi.org/10.1098/rsos.190741

Kaya, N., \& Epps, H. H. (2004). Relationship between color and emotion: a study of college students. College Student Journal, 38(3), 396-406. Retrieved from https://nzdis.org/projects/attachments/299/colorassociation-students.pdf

Lindborg, P., \& Friberg, A. K. (2015). Colour association with music is mediated by emotion: Evidence from an experiment using a CIE Lab interface and interviews. PloS One, 10(12), e0144013. https://doi.org/10.1371/journal.pone.0144013 
Mohr, C., Jonauskaite, D., Dan-Glauser, E. S., Uusküla, M., \& Dael, N. (2018). Unifying research on colour and emotion: Time for a cross-cultural survey on emotion associations with colour terms. In L. W. MacDonald, C. P. Biggam, \& G. V Paramei (Eds.), Progress in colour studies: Cognition, language, and beyond (pp. 209-222). https://doi.org/10.1075/z.217.11moh

Simmons, J. P., Nelson, L. D., \& Simonsohn, U. (2011). False-positive psychology: Undisclosed flexibility in data collection and analysis allows presenting anything as significant. Psychological Science, 22(11), 1359-1366. https://doi.org/10.1177/0956797611417632

Sutton, T. M., \& Altarriba, J. (2016). Color associations to emotion and emotion-laden words: A collection of norms for stimulus construction and selection. Behavior Research Methods, 48(2), 686-728. https://doi.org/10.3758/s13428-015-0598-8 